\title{
The prognostic value of health-related quality-of-life data in predicting survival in glioblastoma cancer patients: results from an international randomised phase III EORTC Brain Tumour and Radiation Oncology Groups, and NCIC Clinical Trials Group study
}

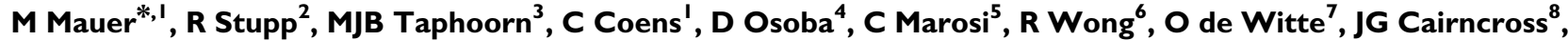
F Efficace', RO Mirimanoff ${ }^{2}$, P Forsyth ${ }^{9}$, MJ van den Bent ${ }^{10}$, M Weller ${ }^{11}$ and A Bottomley ${ }^{1}$

'European Organisation for Research and Treatment of Cancer, Quality of Life Unit, Data Center, Avenue Mounier 83/II, I 200 Brussels, Belgium; ${ }^{2}$ Centre Hospitalier Universitaire Vaudois and University of Lausanne, rue du Bugnon 46, 1011 Lausanne, Switzerland; ${ }^{3}$ Medical Center Haaglanden/ Westeinde Hospital, Lijnbaan 32 Box 432, 250I The Hague, The Netherlands; ${ }^{4}$ QOL consulting, Edendale Court 4939, West Vancouver V7W 3H7, Canada; ${ }^{5}$ Medical University of Vienna, Waehringer Guertel 18-20, 1090 Vienna, Austria; ${ }^{6}$ Hamilton Health Sciences, Juravinski Cancer Center, Concession Street 699, Hamilton, Ontario L8V 5C2, Canada ; ${ }^{7}$ Hopital Universitaire Erasme, Route de Lennik 808, 1070 Brussels, Belgium; ${ }^{8}$ Department of Clinical Neurosciences, University of Calgary and Foothills Hospital, Alberta, Canada; ${ }^{9}$ Tom Baker Cancer Center, University of Calgary, I33I 29th Street N.W., Calgary, AB T2N 4N2, Canada; ${ }^{10}$ Erasmus University Medical Center, Postbus 520 I, 3008 AE Rotterdam, The Netherlands; "'Department of Neurology, University of Tübingen, Hoppe-Seyler-Str. 3, 72076 Tübingen, Germany
\end{abstract}

This is one of the few studies that have explored the value of baseline symptoms and health-related quality of life (HRQOL) in predicting survival in brain cancer patients. Baseline HRQOL scores (from the EORTC QLQ-C30 and the Brain Cancer Module (BN 20)) were examined in 490 newly diagnosed glioblastoma cancer patients for the relationship with overall survival by using Cox proportional hazards regression models. Refined techniques as the bootstrap re-sampling procedure and the computation of $C$-indexes and $R^{2}$-coefficients were used to try and validate the model. Classical analysis controlled for major clinical prognostic factors selected cognitive functioning $(P=0.000 \mathrm{I})$, global health status $(P=0.0055)$ and social functioning $(P<0.000 \mathrm{I})$ as statistically significant prognostic factors of survival. However, several issues question the validity of these findings. $C$-indexes and $R^{2}$-coefficients, which are measures of the predictive ability of the models, did not exhibit major improvements when adding selected or all $\mathrm{HRQOL}$ scores to clinical factors. While classical techniques lead to positive results, more refined analyses suggest that baseline $\mathrm{HRQOL}$ scores add relatively little to clinical factors to predict survival. These results may have implications for future use of $H R Q O L$ as a prognostic factor in cancer patients.

British Journal of Cancer (2007) 97, 302-307. doi:I0.1038/sj.bjc.6603876 www.bjcancer.com

Published online 3 July 2007

(c) 2007 Cancer Research UK

Keywords: HRQOL scores; prognostic factors; glioblastoma

It has become increasingly accepted that, in addition to the traditional assessment of clinical outcomes, health-related qualityof-life (HRQOL) information can play a key role in cancer research and may help in individual patient care (Detmar et al, 2002; Velikova et al, 2004). Patients' self-assessment of HRQOL is now an established end point for treatment comparisons in many cancer disease sites (Bottomley et al, 2003), particularly in advanced disease (Efficace et al, 2004a; Bottomley et al, 2005).

Theoretically, HRQOL outcomes could have various clinical applications, including supporting clinical decision-making by providing the patient's perspective or providing prognostic information. Recent studies have shown that HRQOL parameters can be independent prognostic factors for survival in cancer

*Correspondence: Dr M Mauer; E-mail: Murielle.Mauer@eortc.be Received 2 April 2007; revised II June 2007; accepted II June 2007; published online 3 July 2007 patients (Kramer et al, 2000). If HRQOL parameters are independent predictors of survival, they could be used in daily practice to identify patients who will benefit from a specific intervention; further, it could help to avoid over-treatment of patients who will gain no benefit from, often, toxic and aggressive therapies or to set up more tailored psychosocial intervention programmes aimed at improving patients' HRQOL. Furthermore, they could be used to better stratify patients in future cancer clinical trials, hence better interpreting study outcomes or helping identify critical areas that could help in the selection of key end points for future clinical trials.

Health-related quality-of-life prognostic factor analyses have been carried out on several different cancer populations, including, among others, lung (Herndon et al, 1999; Langendijk et al, 2000; Montazeri et al, 2001; Efficace et al, 2006), oesophageal (Blazeby et al, 2001; Fang et al, 2004), advanced breast (Luoma et al, 2003; Efficace et al, 2004a), and head and neck (de Graeff et al, 2001) 
cancers, highlighting the importance HRQOL scores may have in predicting survival. Only three studies have examined HRQOL and/or cognitive functioning as a prognostic factor in brain cancer (Meyers et al, 2000; Klein et al, 2003; Sehlen et al, 2003). The present study evaluates the prognostic value of HRQOL data collected from a prospective, large-scale international randomised controlled trial, by using various statistical techniques in an attempt to provide robust conclusions on the prognostic value of HRQOL in glioblastoma multiforme patients.

\section{METHODS}

\section{Treatment}

In this international, multicentre study (EORTC trial 26981-22981NCIC CE3), 573 patients with newly diagnosed glioblastoma were randomised to treatment with radiotherapy (RT) only or RT with concomitant temozolomide (TMZ) chemotherapy followed by six cycles of TMZ chemotherapy. Patients were stratified for institution, performance status (WHO performance status 0 or 1 vs 2), age $(<50 v s \geqslant 50$ years) and the extent of the resection at surgery (biopsy only vs debulking surgery/resection). The details on trial conduct and clinical outcome have previously been reported (Hegi et al, 2005; Stupp et al, 2005; Mirimanoff et al, 2006). The trial was approved by the EORTC protocol review committee and the ethics committee of each participating centre. All patients provided written informed consent.

\section{Procedures}

Health-related quality-of-life evaluations The primary end point of the clinical trial was survival, with HRQOL being a secondary end point. Two HRQOL measures were selected for the trial: the EORTC Quality of Life Questionnaire C30 (EORTC QLQ-C30, version 2) (Aaronson et al, 1993) and the EORTC QLQ-Brain Cancer Module (QLQ-BN20) (Osoba et al, 1996). Both tools have robust psychometric properties resulting from rigorous testing and development from their use in several international cancer clinical trials (Bottomley et al, 2003). The EORTC QLQ-C30 is a core measure designed to be supplemented with disease-specific questionnaires. The EORTC QLQ-BN20 was developed specifically in patients with brain cancer. Both instruments were available in the language of all participating patients (Cull et al, 2002).

The EORTC QLQ-C30 measure comprises five functioning scales - physical, role, emotional, cognitive and social; three symptom scales - fatigue, nausea/vomiting and pain; six single item scales dyspnoea, insomnia, appetite loss, constipation, diarrhoea and financial impact; and the overall health/global QOL scale.

The EORTC QLQ-BN20, designed for use with patients undergoing chemotherapy or RT, includes 20 items assessing visual disorders, motor dysfunction, communication deficit, various disease symptoms (e.g. headaches and seizures), treatment toxicities (e.g. hair loss) and future uncertainty.

The items on both measures were scaled and scored using the recommended EORTC procedures (Fayers et al, 2001). Raw scores were transformed to a linear scale ranging from 0 to 100 , with a higher score representing a higher level of functioning or higher level of symptoms. Provided at least half of the items in the scale were completed, the scale score was calculated using only those items for which values existed.

Patients were randomised following surgery and before the start of the RT. Radiotherapy started within 6 weeks after surgery. Valid HRQOL assessments were performed at baseline, before the start of therapy, not more than 1 month before randomisation and not more than 2 weeks after randomisation.

Health-related quality of life was a mandatory aspect of this clinical trial protocol. The protocol stipulated that a responsible nurse, clinician or data manager administered the questionnaire, requesting completion and its return to the EORTC Data Centre. The EORTC guidelines for administering questionnaires were provided, ensuring a standard approach and optimal compliance of HRQOL data collection by all personnel.

\section{Statistical analysis}

For this analysis, only baseline HRQOL scores were used. To minimise the risk of false-positive results, we excluded $a$ priori several HRQOL scales (seven from the QLQ-C30 and two from the brain module) from the analyses. The variables selected to be excluded were not expected to have any prognostic value or alternatively were known to be highly inter-correlated with other scales, thereby not contributing to the model. The final analysis included 17 HRQOL variables: eight (appetite loss, cognitive functioning, emotional functioning, fatigue, physical functioning, global health status, social functioning, insomnia) from the core questionnaire and nine (bladder control, communication deficit, drowsiness, future uncertainty, headaches, motor dysfunction, seizures, visual disorder, weakness of legs) from the brain module.

The Cox proportional hazards regression model (Cox, 1972) with overall survival measured from the time of randomisation as dependent outcome was used for both univariate and multivariate analyses. A Collett's Model Selection approach (Collett, 1994) was used with a level of significance of 0.15 for the univariate screening and stay and entry criterion of 0.05 . The HRQOL scales were included as continuous factors. The model was controlled for the major established prognostic baseline clinical factors (Gorlia et al, in press): age ( $<50 v s \geqslant 50$ years), performance status ( $0 v s 1 v s 2)$, extent of surgery (complete resection $v s$ partial resection $v s$ biopsy only), corticosteroids at entry (yes vs no), mini-mental state examination $\left(<27\right.$ vs 27-30), $O^{6}$-methylguanine-DNA methyltransferase (MGMT) promoter methylation status (yes $v s$ no) as well as randomly assigned therapy. The MGMT status could only be assessed in $36 \%$ of the patients and was shown to be predictive of a favourable treatment effect in patients receiving TMZ chemotherapy (Hegi et al, 2005). The MGMT promoter methylation status was included in the analysis as a stratum with three levels (methylated, unmethylated or missing). The treatment assignment was also included as a stratum (because of the interaction between treatment and MGMT methylation). All other factors were included as covariates.

Validation of the final model was undertaken by use of several refined statistical techniques. The stability of the final model was investigated using a bootstrap re-sampling procedure as proposed by Sauerbrei and Schumacher (1992), applied in the context of HRQOL (Van Steen et al, 2002). This technique generates a number of samples (each of the same size as the original data set), by randomly selecting patients and replacing them before selecting the next patient (i.e. bootstrap resampling). The frequency of inclusion of the HRQOL scores in the Cox PH regression models, including all the selected clinical factors and treatment, fitted to each of these data sets using automatic forward stepwise selection (entry level of $\alpha=0.05$ ), can be considered to be indicative for the importance of the HRQOL factors. This technique was applied to 1000 bootstrap samples.

Deviance residuals from the model with clinical factors were plotted $v s$ the HRQOL scores to explore the relationship between each HRQOL score and the remaining part of the hazard not already explained by clinical factors. Finally, discrimination $C$-indexes and Nagelkerke's $R^{2}$ coefficients were computed to quantify the predictive accuracy of a model. $C$-index is a measure of how well a model is able to rightly predict which patient among a randomly chosen pair of patients will survive longer. Nagelkerke's $R^{2}$-coefficient is a generalisation of the $R^{2}$-coefficient in linear regression, which measures the percentage of variation in the dependent variable accounted for by the predictor variables. It 
assesses the ability of the model to separate between all patient responses based on the predictor variables.

All data analyses were performed using Statistical Analysis Software (SAS) version 9.

\section{RESULTS}

Between June 2000 and March 2002, 573 patients from 85 institutions in 15 countries were randomised to receive either RT alone (286 patients) or RT with TMZ (287 patients). Of these patients, 248 (RT) and 242 (RT plus TMZ) had baseline HRQOL measures completed. In total, 83 (RT) and 86 (RT plus TMZ) had an assessment of their methylation status of the MGMT promoter. The analysis was performed on 490 patients with baseline HRQOL measures that represent $85.5 \%$ of the original sample size.

\section{Clinical and quality-of-life results}

The clinical results have been published (Hegi et al, 2005; Stupp et al, 2005; Mirimanoff et al, 2006). In brief, the study demonstrated that the addition of TMZ to RT for newly diagnosed glioblastoma resulted in a clinically meaningful and statistically significant survival benefit with minimal additional toxicity (unadjusted hazard ratio of 0.63 with a $95 \%$ confidence interval, $0.52-0.75 ; P<0.001$ by the log-rank test). In addition, patients whose tumours had a methylated MGMT gene promoter benefited from TMZ, whereas those who did not have a methylated MGMT promoter derived no or only limited benefit from the addition of chemotherapy. Quality-of-life results have also been published (Taphoorn et al, 2005). Addition of TMZ during and after RT significantly improved survival without a negative effect on HRQOL. Baseline clinical characteristics for patients with a valid baseline HRQOL questionnaire are depicted in Table 1.

The distribution of the baseline clinical characteristics was very similar between patients with a valid baseline HRQOL questionnaire and those without. When comparing patients with and those without available MGMT assessment, imbalances were noticed for the clinical characteristics age, MMSE score, extent of surgery and tumour location. The percentages of patients under 50 years, with an MMSE above 27 or with a unifocal tumour location are higher in patients with a MGMT status. The subgroup of patients with MGMT promoter methylation status assessed was therefore not entirely representative of the whole trial population. A higher proportion of patients with resected tumours had the MGMT status successfully assessed due to the lack of a sufficient amount of tumour tissue in stereotactic biopsies.

\section{Prognostic factor analysis results}

Univariate analysis When controlled for the major prognostic clinical factors, the HRQOL scores that passed the $15 \%$ significance level were cognitive functioning, fatigue, global health status, social functioning, bladder control, headaches and motor dysfunction. Table 2 shows the results of this analysis.

\section{Multivariate analysis}

The Cox multivariate model selected by Collett's Model Selection approach contained cognitive functioning, global health status and social functioning in addition to the selected clinical factors (Table 3). However, the sign of the coefficient related to social functioning was opposite to what was expected, that is, worse social functioning was related to better survival.

\section{Bootstrap re-sampling procedure}

Table 4 presents the results of the bootstrap re-sampling procedure. The inclusion frequencies above $50 \%$ were related to
Table I Baseline clinical characteristics of patients with HRQOL

Baseline characteristics

\begin{tabular}{|c|c|c|c|}
\hline & \multicolumn{2}{|c|}{ Treatment } & \multirow{3}{*}{$\begin{array}{c}\text { Total } \\
(N=490) \\
N(\%)\end{array}$} \\
\hline & $\begin{array}{c}\text { RT } \\
(N=248)\end{array}$ & $\begin{array}{l}\text { RT+TMZ } \\
(N=242)\end{array}$ & \\
\hline & $N(\%)$ & $N(\%)$ & \\
\hline \multicolumn{4}{|l|}{ Age (years) } \\
\hline$<50$ & $72(29.0)$ & $75(31.0)$ & $147(30.0)$ \\
\hline$\geqslant 50$ & $176(71.0)$ & $167(69.0)$ & $343(70.0)$ \\
\hline \multicolumn{4}{|l|}{ Sex } \\
\hline Male & $152(6 \mid .3)$ & I53 (63.2) & $305(62.2)$ \\
\hline Female & $96(38.7)$ & $89(36.8)$ & $185(37.8)$ \\
\hline \multicolumn{4}{|l|}{ MMSE score } \\
\hline$<27$ & 78 (31.5) & $68(28.1)$ & | 46 (29.8) \\
\hline $27-30$ & $167(67.3)$ & $168(69.4)$ & $335(68.4)$ \\
\hline Missing & $3(1.2)$ & $6(2.5)$ & $9(1.8)$ \\
\hline \multicolumn{4}{|l|}{ PFS (WHO) } \\
\hline 0 & 95 (38.3) & $96(39.7)$ & 191 (39.0) \\
\hline I & $120(48.4)$ & $113(46.7)$ & $233(47.6)$ \\
\hline 2 & $33(13.3)$ & $33(13.6)$ & $66(13.5)$ \\
\hline \multicolumn{4}{|l|}{ Corticosteroids at entry } \\
\hline No & $63(25.4)$ & 78 (32.2) & $|4|$ (28.8) \\
\hline Yes & $185(74.6)$ & $164(67.8)$ & $349(71.2)$ \\
\hline \multicolumn{4}{|l|}{ Extent of surgery } \\
\hline Biopsy only & $39(15.7)$ & $38(15.7)$ & $77(15.7)$ \\
\hline Partial resection & $116(46.8)$ & $107(44.2)$ & $223(45.5)$ \\
\hline Total resection & 93 (37.5) & $97(40.1)$ & $190(38.8)$ \\
\hline \multicolumn{4}{|l|}{ Tumour location } \\
\hline Unifocal & $206(83.1)$ & $198(81.8)$ & $404(82.4)$ \\
\hline Multifocal+other & $42(16.9)$ & $44(18.2)$ & $86(17.6)$ \\
\hline \multicolumn{4}{|l|}{ MGMT } \\
\hline Methylated & $38(15.3)$ & $40(16.5)$ & $78(15.9)$ \\
\hline Unmethylated & $48(19.4)$ & $49(20.2)$ & $97(19.8)$ \\
\hline Missing & $162(65.3)$ & $153(63.2)$ & $315(64.3)$ \\
\hline
\end{tabular}

cognitive functioning (83.0\%), global health status (55.2\%) and social functioning $(88.9 \%)$. The frequencies of selection of each possible set of HRQOL scores were very low. The most frequently selected model (cognitive functioning, global health status, social functioning) was selected only $8.8 \%$ of the time. This indicates a high degree of model instability with no single model to be uniformly preferable over all others.

\section{Discrimination index $C$ and Nagelkerke's $R^{2}$ coefficient}

Discrimination $C$-indexes and Nagelkerke's $R^{2}$ coefficients were computed for the model with clinical prognostic factors only and for various models with added HRQOL scores. The $C$-index of the model with only clinical baseline characteristics is $C=0.647$ and the Nagelkerke's $R^{2}$-coefficient is $R^{2}=0.133$. When adding HRQOL scores, $C=0.654$ and $R^{2}=0.177$ for the three selected HRQOL scores and $C=0.647$ and $R^{2}=0.189$ for all HRQOL scores. For the model without clinical factors but with all HRQOL scores, $C=0.604$ and $R^{2}=0.104$.

\section{DISCUSSION}

Glioblastoma, like any other malignant brain tumour, have a considerable impact on the patient's HRQOL, and in spite of 
Table 2 Univariate prognostic factor analyses for survival (controlled for clinical factors)

\begin{tabular}{|c|c|c|c|c|}
\hline \multirow{2}{*}{$\frac{\text { HRQOL scores }^{\mathbf{a}}}{\text { EORTC QLQ-C30 }}$} & \multirow[t]{2}{*}{ Hazard ratio (HR) } & \multicolumn{2}{|c|}{$\begin{array}{l}95 \% \text { confidence } \\
\text { interval }(\mathrm{Cl})\end{array}$} & \multirow[t]{2}{*}{$P$-value } \\
\hline & & & & \\
\hline Appetite loss & 1.017 & 0.967 & 1.069 & 0.5088 \\
\hline Cognitive functioning & 0.919 & 0.887 & 0.953 & $<0.0001$ \\
\hline Emotional functioning & 0.983 & 0.943 & 1.025 & 0.4162 \\
\hline Fatigue & 1.063 & 1.022 & 1.106 & 0.0025 \\
\hline Physical functioning & 0.927 & 0.890 & 0.967 & 0.0004 \\
\hline Global health status & 0.939 & 0.901 & 0.977 & 0.0022 \\
\hline Social functioning & 1.012 & 0.981 & 1.044 & 0.4504 \\
\hline Insomnia & 0.994 & 0.961 & 1.029 & 0.7308 \\
\hline \multicolumn{5}{|l|}{ EORTC QLQ-BN2O } \\
\hline Bladder control & 1.066 & 1.019 & 1.115 & 0.0055 \\
\hline Communication deficit & 1.088 & 1.046 & 1.132 & $<0.0001$ \\
\hline Drowsiness & 1.021 & 0.982 & 1.061 & 0.2967 \\
\hline Future uncertainty & 1.019 & 0.983 & 1.055 & 0.3051 \\
\hline Headaches & 1.024 & 0.984 & 1.065 & 0.2373 \\
\hline Motor dysfunction & 1.092 & 1.048 & 1.139 & $<0.0001$ \\
\hline Seizures & 1.079 & 1.020 & 1.140 & 0.0079 \\
\hline Visual disorder & 1.028 & 0.966 & 1.093 & 0.3857 \\
\hline Weakness of legs & 1.044 & 1.005 & 1.084 & 0.0276 \\
\hline
\end{tabular}

${ }^{\mathrm{a}}$ For every 10 point shift in the scale ranging between 0 and 100 .

Table 3 Multivariate model predicting survival for patients with HRQOL

\begin{tabular}{|c|c|c|c|}
\hline Variables & Hazard ratio (HR) & $\begin{array}{c}95 \% \\
\text { confidence } \\
\text { interval } \\
\quad(\mathrm{Cl})\end{array}$ & $P$-value \\
\hline \multicolumn{4}{|l|}{ Clinical variables } \\
\hline Age $(<50$ vs $\geqslant 50$ years $)$ & 1.274 & 1.0081 .611 & 0.0430 \\
\hline Biopsy vs partial resection & 0.830 & 0.6141 .121 & 0.2246 \\
\hline Biopsy vs total resection & 0.618 & 0.4480 .853 & 0.0034 \\
\hline Performance status ( 0 vs I) & 1.069 & 0.8441 .354 & 0.5803 \\
\hline Performance status ( 0 vs 2 ) & 1.170 & $0.825 \quad 1.659$ & 0.3780 \\
\hline MMSE $(<27$ vs $27-30)$ & 0.679 & 0.5360 .861 & 0.0014 \\
\hline Corticosteroids at entry (no vs yes) & 1.583 & 1.2422 .016 & 0.0002 \\
\hline \multicolumn{4}{|l|}{ HRQOL scores ${ }^{\mathrm{a}}$} \\
\hline Cognitive functioning & 0.918 & 0.8780 .959 & 0.0001 \\
\hline Global health status & 0.929 & 0.8820 .979 & 0.0055 \\
\hline Social functioning & 1.090 & 1.0461 .137 & $7<0.000$ । \\
\hline
\end{tabular}

a For every 10 point shift in the scale ranging between 0 and 100

important progress due to the addition of chemotherapy to RT and surgery, as a rule most glioblastoma patients will eventually suffer from tumour relapse. With such a poor prognosis, evaluation of patients' HRQOL before and during treatments becomes important. In this study, we explored if HRQOL data provide reliable and useful prognostic information. Our study used 490 glioblastoma patients, controlled for major prognostic clinical factors, and attempted to overcome limitations of past studies by using a larger sample size of homogeneously treated patients and sophisticated statistical methodology.

Previous studies in brain tumour patients showed that HRQOL factors and/or cognitive functioning were statistically significant factors in prediction models for groups of patients, comparable to our classical analysis. For example, Sehlen et al (2003) examined HRQOL in 153 patients with either malignant astrocytoma or brain metastases. Using the Functional Assessment of Cancer Therapy General HRQOL measure (Cella et al, 1993), they found two variables, that is, 'living with a spouse' and the FACT-G total score to predict survival. Two other studies demonstrated objective
Table 4 Inclusion frequencies in the bootstrap re-sampling procedure

\begin{tabular}{lccc}
\hline Variable & $\begin{array}{c}\text { Inclusion } \\
\text { (\%) }\end{array}$ & $\begin{array}{c}\text { Pos. incl. } \\
\text { (\%) }\end{array}$ & $\begin{array}{c}\text { Neg. incl. } \\
\text { (\%) }\end{array}$ \\
\hline Age (<50 vs ₹50) & 100.0 & 96.9 & 3.1 \\
Partial resection vs biopsy & 100.0 & 14.0 & 86.0 \\
Total resection vs biopsy & 100.0 & 1.3 & 98.7 \\
Performance status ( I vs 0) & 100.0 & 75.9 & 24.1 \\
Performance status (2 vs 0) & 100.0 & 67.9 & 32.1 \\
MMSE (<27 vs 27-30) & 100.0 & 0.3 & 99.7 \\
Corticosteroids at entry (yes vs no) & 100.0 & 99.9 & 0.1 \\
Appetite loss & 8.6 & 5.9 & 2.7 \\
Cognitive functioning & 83.0 & 0.0 & 83.0 \\
Emotional functioning & 7.5 & 6.6 & 0.9 \\
Fatigue & 8.6 & 7.7 & 0.9 \\
Physical functioning & 6.6 & 2.8 & 3.8 \\
Global health status /QoL & 55.2 & 0.0 & 55.2 \\
Social functioning & 88.9 & 88.9 & 0.0 \\
Insomnia & 20.5 & 0.1 & 20.4 \\
Bladder control & 24.2 & 24.2 & 0.0 \\
Communication deficit & 11.9 & 0.9 & 11.0 \\
Drowsiness & 5.2 & 2.3 & 2.9 \\
Future uncertainty & 27.4 & 27.3 & 0.1 \\
Headaches & 8.9 & 7.9 & 1.0 \\
Motor dysfunction & 20.0 & 20.0 & 0.0 \\
Seizures & 13.4 & 13.1 & 0.3 \\
Visual disorder & 9.4 & 5.8 & 3.6 \\
Weakness legs & 20.6 & 1.0 & 19.6 \\
\hline Postive insion is & &
\end{tabular}

Positive inclusion is any inclusion of the factor in a model with a positive coefficient. Negative inclusion is any inclusion of the factor in a model with a negative coefficient.

cognitive functioning to have prognostic significance, both in newly diagnosed and in recurrent high-grade glioma (Meyers et al, 2000; Klein et al, 2003). Meyers et al (2000) examined HRQOL and cognitive functioning in 80 patients with recurrent malignant glioma or anaplastic astrocytoma, at baseline, before treatment in phase I and II trials. Health-related quality of life was undertaken with the FACT-BR module, along with other neuropsychological tests. Health-related quality-of-life scores did not predict survival, but cognitive functioning was a significant predictor of survival. It is difficult to compare these findings with our results, given the different measures that were used, along with their sample being relatively small. In addition, the Phase I/II setting of Meyers et al is likely to be considerably different (higher expectations and discounting toxicities) to that of a large phase III trial (Cheng et al, 2000). Klein et al (2003) explored cognitive functioning along with activities of daily living in 68 newly diagnosed high-grade glioma patients. Cognitive functioning had prognostic value, but only in a subsample of older patients. However, it is unclear to what extent studies on such small samples can be relied on for providing definitive conclusions. It is also difficult to make comparisons between our trial and Klein et al. due to the different HRQOL measures employed.

There are several issues questioning the validity and the reliability of the results obtained by classical techniques. Some of them are well known, such as the large number of HRQOL scales and the intercorrelation of these HRQOL scales. It makes the selection of a particular set of HRQOL scores quite difficult as various sets of HRQOL scores may predict equally survival when added to clinical factors. It may also lead to models difficult to interpret (with worst HRQOL status associated with longer survival) as some HRQOL factors may enter the model just as corrections for others. In addition, as HRQOL scores are analysed as continuous factors, the results could be influenced by a few 'outliers', that is, patients with some very bad HRQOL scores but who actually survived long or vice versa. Furthermore, the residuals plots also suggested a high variability in duration of survival among patients who have a same level of the HRQOL scores. 
$C$-indexes and $R^{2}$-coefficients are thought to better assess the potential benefit of using baseline HRQOL scores in addition to the clinical factors to predict survival in clinical practice and research. In our study, the calculated coefficients did not exhibit major improvement when adding selected or all HRQOL scores to clinical factors, suggesting that baseline HRQOL scores in the end add relatively little to known clinical factors to predict survival and cannot be used alone in outcome prediction.

Care needs to be taken when interpreting the results of our study, given our study had limitations, particularly as this was an exploratory analysis. Also, while 490 patients represent a considerable sample, other data sets are required to validate these findings.

In summary, while traditional methods of analysis suggest HRQOL data are prognostic, more detailed analysis revealed these findings may not be as reliable as expected. Further research should investigate the use of HRQOL with more sophisticated techniques to obtain reliable results.

The investigation of the prognostic value of HRQOL data is a challenging and ongoing research area. Further research could also investigate the prognostic value of changes from baseline in HRQOL rather than baseline values and should investigate why HRQOL parameters might be of value in one setting but not another. Furthermore, the reason for this association between HRQOL data and survival is unclear. Some hypotheses have been proposed to explain the mechanisms underlying the association. Patients' HRQOL scores might reflect an early perception of the severity of the disease in a more accurate way than conventional prognostic indices. In this case, patients who report worse HRQOL scores are the ones with a worse underlying disease. This hypothesis does not imply a true causative relationship between HRQOL parameters and survival. On the other hand, it is also possible that a better HRQOL score (which reflects a better physical and psychological state) could somehow have a positive effect on the disease process by, for example, slowing tumour progression. This causative explanation could be supported by some intervention studies which have shown that psychosocial support improved both psychological well-being and survival time. Coates et al (2000) assumed that, if the mechanism underlying the association between HRQOL and survival is causative, one should expect to see HRQOL parameters being prognostic of clinical outcomes, not only in patients with metastatic disease, but also at an earlier stage of the disease. Given this assumption, and the fact that their study did not find a correlation between HRQOL parameters and disease-free survival in their nonmetastatic breast cancer population, the authors argued in favour of the explanation that HRQOL scores reflect a more accurate perception of the severity of the underlying illness. The results of Efficace et al $(2004 a, b)$ also seem to support this view. Hence, it would be possible to speculate that for early stage disease, clinical examinations (such as performance status or tumour staging) are more likely to supersede patients' self-reported HRQOL scores in predicting survival. However, more studies are required to definitively exclude any possible causative relationship with survival.

\section{ACKNOWLEDGEMENTS}

The data for this analysis were collected by EORTC Brain Tumor Group (BTG), Radiation Oncology Group (ROG) and National Cancer Institute of Canada (NCIC) investigators. This initial clinical trial was conducted by the European Organisation for Research and Treatment of Cancer (EORTC). This research study was supported in part by grants from the National Cancer Institute (5U10CA11488-30 through 5U10CA11488-34) and by the EORTC BTG. We thank all the patients who kindly agreed to participate in this study and all the investigators for their involvement. We kindly thank Dr L Collette at the EORTC for providing support for applying the refined statistical techniques, specifically the computation of $C$-indexes to our data.

\section{REFERENCES}

Aaronson NK, Ahmedzai S, Bergman B, Bullinger M, Cull A, Duez NJ, Filiberti A, Flechtner H, Fleishman SB, de Haes JC (1993) The European Organization for Research and Treatment of Cancer QLQ-C30: a qualityof-life instrument for use in international clinical trials in oncology. J Natl Cancer Inst 85: 365-376

Blazeby JM, Brookes ST, Alderson D (2001) The prognostic value of quality of life scores during treatment for oesophageal cancer. Gut 49: 227-230

Bottomley A, Flechtner H, Efficace F, Vanvoorden V, Coens C, Therasse P, Velikova G, Blazeby J, Greimel E (2005) Health related quality of life outcomes in cancer clinical trials. Eur J Cancer 41: 1697-1709

Bottomley A, Vanvoorden V, Flechtner H, Therasse P (2003) The challenge and achievements of implementation of quality of life research in cancer clinical trials. Eur J Cancer 39: 275-285

Cella DF, Tulsky DS, Gray G, Sarafian B, Linn E, Bonomi A, Silberman M, Yellen SB, Winicour P, Brannon J (1993) The Functional Assessment of Cancer Therapy scale: development and validation of the general measure. J Clin Oncol 11(3): 570-579

Cheng JD, Hitt J, Koczwara B, Schulman KA, Burnett CB, Gaskin DJ, Rowland JH, Meropol NJ (2000) Impact of quality of life on patient expectations regarding phase I clinical trials. J Clin Oncol 18(2): 421 -428

Coates AS, Hurny C, Peterson HF, Bernhard J, Castiglione-Gertsch M, Gelber RD, Goldhirsch A (2000) Quality-of-life scores predict outcome in metastatic but not early breast cancer. International Breast Cancer Study Group. J Clin Oncol 18(22): 3768-3774

Collett D (1994) Modelling Survival Data in Medical Research. Chapman \& Hall: London

Cox DR (1972) Regression models and life tables. J Royal Stat Soc 34: 187-220

Cull A, Sprangers M, Bjordal K, Aaronson N (2002) EORTC Quality of Life Group Translation Procedure. EORTC Publications: Brussels, ISBN: 2-930064-28-5 de Graeff A, de Leeuw JR, Ros WJ, Hordijk GJ, Blijham GH, Winnubst JA (2001) Sociodemographic factors and quality of life as prognostic indicators in head and neck cancer. Eur J Cancer 37: 332-339

Detmar SB, Muller MJ, Schornagel JH, Wever LD, Aaronson NK (2002) Health-related quality-of-life assessments and patient-physician communication: a randomized controlled trial. JAMA 288(23): 3027-3034

Efficace F, Biganzoli L, Piccart M, Coens C, Van Steen K, Cufer T, Coleman RE, Calvert HA, Gamucci T, Twelves C, Fargeot P, Bottomley A (2004a) Baseline health-related quality-of-life data as prognostic factors in a phase III multicentre study of women with metastatic breast cancer. Eur J Cancer 40(7): $1021-1030$

Efficace F, Bottomley A, Smit EF, Lianes P, Legrand C, Debruyne C, Schramel F, Smit HJ, Gaafar R, Biesma B, Manegold C, Coens C, Giaccone G, Van Meerbeeck J (2006) Is a patient's self-reported health-related quality of life a prognostic factor for survival in non-small-cell lung cancer patients? A multivariate analysis of prognostic factors of EORTC study 08975. Ann Oncol 17(11): 1698-1704

Efficace F, Therasse P, Piccart MJ, Coens C, van Steen K, WelnickaJaskiewicz M, Cufer T, Dyczka J, Lichinitser M, Shepherd L, de Haes H, Sprangers MA, Bottomley A (2004b) Health-related quality of life parameters as prognostic factors in a nonmetastatic breast cancer population: an international multicenter study. J Clin Oncol 22(16): $3381-3388$

Fang FM, Tsai WL, Chiu HC, Kuo WR, Hsiung CY (2004) Quality of life as a survival predictor for esophageal squamous cell carcinoma treated with radiotherapy. Int J Radiat Oncol Biol Phys 58: 1394-1404

Fayers P, Aaronson N, Bjordal K, Groenvold M, Curran D, Bottomley A (2001) EORTC QLQ-C30 Scoring Manual. EORTC Publications: Brussels, ISBN: 2-930064-22-6 
Gorlia T, van den Bent MJ, Hegi M. Nomograms for predicting survival of patients with newly diagnosed glioblastoma multiforme: a prognostic factor analysis of EORTC/NCIC trial 26981-22981/CE. Clin Oncol (in press)

Hegi ME, Diserens AC, Gorlia T, Hamou MF, de Tribolet N, Weller M, Kros JM, Hainfellner JA, Mason W, Mariani L, Bromberg JE, Hau P, Mirimanoff RO, Cairncross JG, Janzer RC, Stupp R (2005) MGMT gene silencing and benefit from temozolomide in glioblastoma. $N$ Engl J Med 352(10): $997-1003$

Herndon JE, Fleishman S, Kornblith AB, Kosty M, Green MR, Holland J (1999) Is quality of life predictive of the survival of patients with advanced non-small cell lung carcinoma? Cancer 5 85(2): $333-340$

Klein M, Postma TJ, Taphoorn MJ, Aaronson NK, Vandertop WP, Muller M, van der Ploeg HM, Heimans JJ (2003) The prognostic value of cognitive functioning in the survival of patients with high-grade glioma. Neurology 61(12): 1796-1798

Kramer JA, Curran D, Piccart M, de Haes JC, Bruning P, Klijn J, Van Hoorebeeck I, Paridaens R (2000) Identification and interpretation of clinical and quality of life prognostic factors for survival and response to treatment in first-line chemotherapy in advanced breast cancer. Eur J Cancer 36: 1498 - 1506

Langendijk H, Aaronson NK, de Jong JM, ten Velde GP, Muller MJ, Wouters M (2000) The prognostic impact of quality of life assessed with the EORTC QLQ-C30 in inoperable non-small cell lung carcinoma treated with radiotherapy. Radiother Oncol 55: 19-25

Luoma ML, Hakamies-Blomqvist L, Sjöström J, Pluzanska A, Ottoson S, Mouridsen H, Bengtsson NO, Bergh J, Malmstrom P, Valvere V, Tennvall L, Blomqvist C (2003) Prognostic value of quality of life scores for time to progression (TTP) and overall survival time (OS) in advanced breast cancer. Eur I Cancer 39(10): 1370 - 1376

Meyers CA, Hess KR, Yung WK, Levin VA (2000) Cognitive function as a predictor of survival in patients with recurrent malignant glioma. J Clin Oncol 18(3): 646-650

Mirimanoff RO, Gorlia T, Mason W, Van den Bent MJ, Kortmann RD, Fisher B, Reni M, Brandes AA, Curschmann J, Villa S, Cairncross G,
Allgeier A, Lacombe D, Stupp R (2006) Radiotherapy and temozolomide for newly diagnosed glioblastoma: recursive partitioning analysis of the EORTC 26981/22981-NCIC CE3 phase III randomized trial. J Clin Oncol 24(16): $2563-2569$

Montazeri A, Milroy R, Hole D, McEwen J, Gillis CR (2001) Quality of life in lung cancer patients: as an important prognostic factor. Lung Cancer 31(2-3): $233-240$

Osoba D, Aaronson NK, Muller M, Sneeuw K, Hsu MA, Yung WK, Brada M, Newlands E (1996) The development and psychometric validation of a brain cancer quality-of-life questionnaire for use in combination with general cancer-specific questionnaires. Qual Life Res 5(1): 139-150

Sauerbrei W, Schumacher M (1992) A bootstrap resampling procedure for model building: application to the Cox regression model. Stat Med 11: 2093-2109

Sehlen S, Lenk M, Hollenhorst H, Schymura B, Aydemir U, Herschbach P, Duhmke E (2003) Quality of life (QoL) as predictive mediator variable for survival in patients with intracerebral neoplasma during radiotherapy. Onkologie 26(1): 38-43

Stupp R, Mason WP, van den Bent MJ, Weller M, Fisher B, Taphoorn MJ, Belanger K, Brandes AA, Marosi C, Bogdahn U, Curschmann J, Janzer RC, Ludwin SK, Gorlia T, Allgeier A, Lacombe D, Cairncross JG, Eisenhauer E, Mirimanoff RO (2005) Radiotherapy plus concomitant and adjuvant temozolomide for glioblastoma. N Engl J Med 352(10): 987-996

Taphoorn MJ, Stupp R, Coens C, Osoba D, Kortmann R, van den Bent MJ, Mason W, Mirimanoff RO, Baumert BG, Eisenhauer E, Forsyth P, Bottomley A (2005) Health-related quality of life in patients with glioblastoma: a randomized controlled trial. Lancet Oncol 6(12): 937-944

Van Steen K, Curran D, Kramer J, Molenberghs G, Van Vreckem A, Bottomley A, Sylvester R (2002) Multicollinearity in prognostic factor analyses using the EORTC QLQ-C30: identification and impact on model selection. Stat Med 21(24): 3865-3884

Velikova G, Booth L, Smith AB, Brown PM, Lynch P, Brown JM, Selby PJ (2004) Measuring quality of life in routine oncology practice improves communication and patient well-being: a randomized controlled trial. J Clin Oncol 22(4): 714-724 